\title{
Malaria Parasitaemia among patients attending General Hospital Minna, North Central Nigeria
}

\author{
Eke, S. S. ${ }^{1 *}$, Omalu, I. C. J. ${ }^{1}$, Olayemi, I. K. ${ }^{1}$, Egwim, E. C. ${ }^{2}$, Hassan, S. C. ${ }^{3}$, \\ Otuu, C. A. ${ }^{1}$, Boyi, A. A. ${ }^{1}$ and Abdullahi, M. ${ }^{1}$ \\ ${ }^{1}$ Department of Animal Biology, Federal University of Technology, Minna, Niger State, Nigeria. \\ ${ }^{2}$ Department of Biochemistry, Federal University of Technology, Minna, Niger State, Nigeria. \\ ${ }^{3}$ Department of Zoology, Nasarawa State University Keffi, Nasarawa State, Nigeria.
}

*Corresponding author. Email: ekesamuel2012@gmail.com

Copyright (C) 2018 Eke et al. This article remains permanently open access under the terms of the Creative Commons Attribution License 4.0, which permits unrestricted use, distribution, and reproduction in any medium, provided the original work is properly cited.

Received 1st May, 2018; Accepted 6th June, 2018

\begin{abstract}
Malaria is one of the most widespread parasitic infections globally and is a major cause of mortality, particularly in regions of high malaria endemicity. This study was carried out to investigate the prevalence of malaria infection and parasitaemia among patients attending General Hospital, Minna, North Central Nigeria. A total of one thousand and seventy-one $(1,071)$ individuals within the study area were enrolled for the study from the month of June, 2016 to May, 2017. Malaria parasitaemia was carried out using thick and thin blood films. Parasites counts were reported per 800 white blood cells. The ages of the individuals recruited for the study ranged from 0 to 51 years of age. Out of the $1,071(100 \%)$ blood samples collected, $741(69.19 \%)$ were positive for malaria parasitaemia of which $74(9.99 \%), 134$ $(18.08 \%), 176(23.75 \%)$ and $357(48.18 \%)$ individuals scored malaria parasite densities of +++, ++, + and scanty had parasites in their blood stream respectively. Statistically, there was a significant difference $(P<0.05)$ between malaria parasitaemia and age. On the other hand, of the 1071 (100\%) blood samples collected and screened, there were 607 (56.68\%) males and 464 (43.32\%) females recruited for the study of which 404 (54.52\%) of the total males sampled were infected while $337(45.48 \%)$ of the total females sampled were infected. There was no significant difference $(P>0.05)$ between the degree of malaria parasitaemia in relation to gender (sex). The high prevalence of malaria parasitaemia among patients attending General Hospital, Minna, North Central Nigeria highlights the need for more efforts to be targeted at controlling malaria among individuals as this will ultimately lead to a reduction in the incidence of malaria in Minna and Nigeria at large.
\end{abstract}

Key words: Malaria, parasitaemia, Plasmodium falciparum.

\section{INTRODUCTION}

Arthropod-borne diseases remain a major public health issue in the resource-constrained settings. Since immemorial, mosquitoes are closely associated with mankind and play an important role in the transmission of many dreadful diseases like malaria, filariasis, Japanese encephalitis, dengue and yellow fever (Karunamoorthi and Sabesan, 2013). Global medical reports have shown that mosquito-borne diseases are responsible for significant human morbidity and mortality throughout the world. Snow et al. (2005) and Snow and Marsh, (2002) reported that the magnitude of malaria in terms of morbidity and mortality in humans makes it a major public health problem in tropical and sub-tropical countries. It is estimated that about 300 to 660 million cases of malaria occur every year, of whom around 90 per cent are in sub-Saharan Africa.

Malaria, a disease mostly caused by Plasmodium falciparum, is a major public health problem. The global burden is 207 million malaria cases every year resulting into 627,000 deaths (WHO, 2013), sub-Saharan Africa being the most affected region. In Nigeria, malaria still remains a major health problem with about two-thirds of the population living in malarious areas (WHO, 2014). 
Malaria has been around for thousands of years, and is still a major problem today. Despite efforts to eradicate malaria over the past 100 years, 149 to 274 million cases and 537,000 to 907,000 deaths from malaria occur in subSaharan Africa each year (Goodman et al., 2000). Malaria is caused by microorganisms belonging to the genus Plasmodium, and can infect reptiles, birds and mammals. Of the more than 100 Plasmodium species, four of these infect humans. The transmission of the disease from one human to another involves mosquitoes of the genus Anopheles (WHO, 2011). Anopheles gambiae is the principal vector of malaria in sub-Saharan Africa (Gillies and Coetzee, 1987; Sinka et al., 2010), where more than $90 \%$ of the World's clinical cases are recorded (Sachs and Malaney, 2002). According to recent World Health Organisation's reports and statistics, malaria threatens the life and health status of the world's human population; resulting in as much as 600 million clinical attacks and an estimated one million deaths annually. The disproportionately high intensity of malaria transmission in sub-Saharan Africa is due to the widespread distribution and high vectorial capacity of the primary vector, i.e., $A$. gambiae in the region (Hay et al., 2008). Studies have established this anopheline species as one of the most efficient transmitter of Plasmodium parasites in the world. The epidemiological success of $A$. gambiae is largely dependent on its highly dynamic ecological behaviour (White et al., 2011), that have evolved over a long time to take advantage of certain tropical clement weather conditions that promote mosquito proliferation and human/vector contact.

The administration of blood to a patient is potentially a lifesaving procedure and the demand for blood has greatly increased over the years (Ekwunife et al., 2011). Transfusion therapy although a very important form of treatment in humans, it may be the only option for survival for many patients. Although this therapy helps to save lives, blood can nonetheless be a vehicle for transmission of infections including parasitic diseases (Agboola et al., 2010). Malaria infection has become of more interest to blood banking and blood transfusion based on discoveries that malaria infection may cause methaemoglobinaemia, as haemoglobin taken up by the parasites into their acid food vacuole leads to the spontaneous oxidation of ferrous $(\mathrm{Fe} 2+)$ to ferric $(\mathrm{Fe} 3+)$ iron.

This survey was undertaken to assess the prevalence of Plasmodium falciparum malaria parasitaemia among patients attending general hospital Minna, Niger State, Nigeria. It is envisaged that data derived from the study would enhance the development of malaria control strategies in this area of stable malaria transmission.

\section{MATERIALS AND METHODS}

\section{Study area}

The study was conducted in Minna, Niger State, Nigeria. It is bounded to the north by Kebbi, Sokoto and Kaduna, Federal Capital Territory (FCT) to the East, Benin Republic to the West, Kwara and Kogi to the South. The metropolis spreads across two Local Government Areas namely: Bosso and Chanchaga LGA. The mean annual rainfall of Minna is $1334 \mathrm{~mm}$ with August and September recording the highest monthly rainfall of about $300 \mathrm{~mm}$. the highest monthly temperature is recorded in March with an average daily temperature of $30^{\circ} \mathrm{C}$ and the lowest in August at about $22^{\circ} \mathrm{C}$. Minna has a tropical wet and dry climate with a pronounced dry season.

\section{Study population and blood collection}

A total of one thousand and seventy-one $(1,071)$ subjects comprising of males and females cut across the different health districts of the General Hospital, Minna, Niger State were sampled for malaria screening. Safety procedures were adopted in the collection of finger-prick blood samples by swabbing the area with $70 \%$ alcohol and allow to air dry before the collection begins. The criteria for inclusion in the study were fever or a history of fever in the 24 to 48 hours preceding presentation. The blood collected was transported to the laboratory in heparin bottles at $4^{\circ} \mathrm{C}$ for analysis. Peripheral blood was collected on $1.5 \times 7.0$ $\mathrm{cm}$ strips of Whatman (Brentford, United Kingdom) $3 \mathrm{~mm}$ filter paper and in heparinised bottles. The strips werethen allowed to be air-dried and kept in plastic bags until use.

\section{Estimation of parasite density}

Traditionally, malaria is diagnosed by the demonstration of the organism in Giemsa stained thick smears of peripheral blood, and the different species are distinguished by their appearance in infected erythrocytes (Rubin and Faber, 1994). The technique is capable of accurate and reliable diagnosis, but only when performed by skilled microscopists using defined protocols (Gray et al., 2007) which presents major obstacles for the remote and poor areas of the world.

Thick and thin films of the blood samples were prepared. Thin blood film was fixed with methanol and all the blood films was stained with $3 \%$ Giemsa stain of $\mathrm{pH} 7.0$ for 30 min as reported by Cheesbrough, 2000. Blood films were examined microscopically using x100 (oil immersion) objectives as described by Cheesbrough 2000 and WHO 2000. The thick films were used to determine the parasite densities while thin films were used to identify the parasite species and infective stages. Parasite density per microliter of blood was approximated or estimated from the thick film by taking the number of leucocytes per microliter of blood as 8,000 and expressed as follows:

Parasite density $/ \mathrm{uL}=\frac{\text { Parasite count } \mathrm{x} 8000}{\text { No of } \mathrm{WBC} \text { counted }}$ 
Table 1. Malaria parasitaemia in relation to age.

\begin{tabular}{lccccc}
\hline \multirow{2}{*}{ Age (Years) } & \multicolumn{3}{c}{ Malaria parasite density test } & \multirow{2}{*}{ Total malaria parasite } \\
\cline { 2 - 4 } & $\mathbf{+ + +}$ & $+\boldsymbol{+ +}$ & $\boldsymbol{+}$ & Scanty & $99(71.22)$ \\
$0-5$ & $0(0.00)$ & $12(12.12)$ & $32(32.32)$ & $55(55.56)$ & $65(61.90)$ \\
$6-10$ & $3(4.62)$ & $24(36.92)$ & $10(15.38)$ & $28(43.08)$ & $78(67.24)$ \\
$11-15$ & $8(10.26)$ & $11(14.10)$ & $13(16.67)$ & $46(58.97)$ & $68(64.15)$ \\
$16-20$ & $11(16.18)$ & $9(13.23)$ & $17(25.00)$ & $31(45.59)$ & $87(74.36)$ \\
$21-25$ & $9(10.34)$ & $27(31.03)$ & $12(13.79)$ & $39(44.83)$ & $74(67.27)$ \\
$26-30$ & $5(6.76)$ & $8(10.82)$ & $26(35.14)$ & $35(47.30)$ & $50(83.33)$ \\
$31-35$ & $3(6.00)$ & $7(14.00)$ & $13(26.00)$ & $27(54.00)$ & $51(62.96)$ \\
$36-40$ & $7(13.73)$ & $11(21.57)$ & $12(23.53)$ & $21(41.18)$ & $81(71.05)$ \\
$41-45$ & $15(18.52)$ & $8(9.88)$ & $23(28.39)$ & $35(43.21)$ & $45(75.00)$ \\
$46-50$ & $9(20.00)$ & $10(22.22)$ & $7(15.56)$ & $19(42.22)$ & $43(67.19)$ \\
$51-55$ & $4(9.30)$ & $7(16.28)$ & $11(25.58)$ & $21(48.84)$ & $741(69.19)$ \\
Total & $74(9.99)$ & $134(18.08)$ & $176(23.75)$ & $357(48.18)$ & \\
\hline
\end{tabular}

$X^{2}$ Call $=0.9358 ; X^{2} \mathrm{Tab}=7.81 ; \mathrm{df}=3 ; \mathrm{P}<0.05$.

Plasmodium parasitaemia was graded as follows:

$\leq 500 \mu / L=(+) ; 501 \mu / L-10000 \mu / L=(++)$ and $\geq 10,001 \mu / L=$ $(+++)$.

\section{Laboratory procedures}

Thick and thin blood films were prepared from finger prick blood samples and stained with $10 \%$ Giemsa for $30 \mathrm{~min}$ and examined by immersion oil microscopy with $1,000 \times$ magnification. A slide was declared negative after examining 200 high power fields without parasites. Each slide was read independently and blindly by two certified laboratory microscopists. Parasitaemia was quantified per 200 white blood cells (thick smear) or in case of very high parasitaemia per 1,000 erythrocytes (thin film). Slides with discrepant results were read again by a third reader and the median parasitaemia taken as the final result (Greenwood and Armstrong, 1991).

\section{Ethical approval and clearance}

The aim and objectives of the study was discussed with the Various Ministries of Health (Hospitals Management Boards) in Minna, Niger State. Permission to carry out the study was obtained from these stakeholders. The written consent forms and participant information forms were kept separately from the data collection tools.

\section{Statistical analysis}

Statistical analyses were conducted using SPSS (version 20.0) software. Comparisons between populations were made using Chi-Square analysis. An alpha value of $<0.05$ denoted a statistically significant difference.

\section{RESULTS}

\section{Malaria parasitaemia in relation to age}

Results on age distribution as they relate to malaria parasitaemia are presented in Table 1. Out of the 1,071 (100\%) blood samples collected, 741 (69.19\%) were positive for malaria parasitaemia of which $74(9.99 \%), 134$ $(18.08 \%), 176(23.75 \%)$ and $357(48.18 \%)$ individuals scored malaria parasite densities of,,++++++ and scanty. Malaria parasite density with scanty parasites had the highest density (parasitaemia) of $357(48.18 \%)$ followed by $(+)$ with $176(23.75 \%)$ while +++ malaria density had the least parasite density of $74(9.99 \%)$. On the age group, the highest parasitaemia was recorded among 31 to 35 years age group with parasite density of $50(83.33 \%)$ while the least was $65(61.90 \%)$ recorded among 6 to 10 years age group. Statistically, there was a significant difference $(P<0.05)$ between malaria parasitaemia and age as shown in Table 1.

\section{Malaria parasitaemia in relation to sex}

The results on sex distribution as relate to malaria parasitaemia infection are presented in Table 2. Out of the $1071(100 \%)$ blood samples collected and screened, there were $607(56.68 \%)$ males and $464(43.32 \%)$ females recruited for the study of which $404(54.52 \%)$ of the total males sampled were infected while $337(45.48 \%)$ of the total females sampled were infected. Malaria parasite density with +++ parasitaemia had the highest density in males than in females with parasite density of $43(58.10 \%)$ and $31(41.89 \%)$ respectively. Statistically, there was no significant difference $(P>0.05)$ between the degree of malaria parasitaemia in relation to gender (sex) as shown in Table 2. 
Table 2. Degree of malaria parasitaemia in relation to sex.

\begin{tabular}{lccc}
\hline \multirow{2}{*}{ Degree of malaria Parasitaemia } & \multicolumn{2}{c}{ SEX } & \multirow{2}{*}{ Total malaria parasitaemia } \\
\cline { 2 - 3 } & Male & Female & $176(23.75)$ \\
++ & $93(52.84)$ & $83(47.16)$ & $134(18.08)$ \\
++ & $76(56.72)$ & $58(43.28)$ & $74(9.99)$ \\
+++ & $43(58.10)$ & $31(41.89)$ & $357(48.18)$ \\
Scanty & $192(53.78)$ & $165(46.22)$ & $741(69.19)$ \\
Total & $404(54.52)$ & $337(45.48)$ & \\
\hline
\end{tabular}

$\mathrm{X}^{2}$ Call $=0.9358 ; \mathrm{X}^{2} \mathrm{Tab}=7.81 ; \mathrm{df}=3 ; \mathrm{P}<0.05$.

\section{DISCUSSION}

Malaria is one of the most widespread infections globally and is a major cause of mortality, particularly in regions of high malaria endemicity. This study was carried out between June, 2016 to May, 2017, to investigate the prevalence of malaria infection and parasitaemia among patients attending General Hospital, Minna, North Central Nigeria.

In this study, a high prevalence of $69.19 \%$ malaria parasitamia was observed. This rate though higher than that observed by Erhabor and colleagues in the Niger Delta region of Nigeria as well as by other researchers in Ghana (Erhabor et al., 2007; Muntaka and Opoku-Okrah, 2013) is quite similar to that observed in other parts of Nigeria with rates ranging from 51.5 to $77.4 \%$ prevalence of malaria parasitaemia among blood donors (Epidi et al., 2008; Ekwunife et al., 2011; Badger-Emekaet al., 2013; Mbanugo and Emenalo, 2004). The findings of this study is also higher than prevalence of 28 and $10.2 \%$ respectively observed by Agboola et al. (2010) in Lagos University Teaching Hospital (LUTH) and by Erhabor et al. (2007) in Port Harcourt, Nigeria. Other similar studies carried out around the country including a study carried out in the South East of Nigeria showed a prevalence rate of $40.9 \%$ (Uneke et al., 2006). In Abakaliki metropolis, Epidi et al. (2008) in their work obtained malaria prevalence of (51.5\%) among their blood donors. Similarly, Ekwunife et al. (2011) reported an alarming high rate $(74.1 \%)$ of malaria infection among blood donors in Onitsha urban area. The high prevalence of malaria parasitaemia in this area could be due to the effects of climatic factors such as temperature, humidity and rainfall which regulate the biology and development of both mosquito and parasite, as well as the behavioural attitude of the inhabitants of the areas. The study areas have climatic factors and environmental conditions which favour breeding of malaria vectors and parasites resulting in the probable abundance of malaria vectors.

The highest infection rate was recorded among 31 to 35 years age group. This is similar to the work of Muntaka and Opoku-Okrah (2013) and might be a reflection of the risk taking behaviour of adolescents and young adults who are also less likely to pay much attention to malaria control practices such as sleeping under insecticides treated bed nets, wearing of protective sleep wear. This is contrary to a study conducted in the south Eastern part of the country, where the prevalence of malaria parasitaemia decrease with increasing age, and individuals aged 20 to 25 years were most infected (Uneke et al., 2006).

Based on sex, higher malaria parasitaemia of $54.52 \%$ was recorded among males. This was not in agreement with the study of Wariso and Oboro (2015) in Port Harcourt, South-South, Nigeria who recorded a higher infection rate of $97.5 \%$ among males. The reasons for the observed sex differences are not farfetched as some of the males exposed themselves more than females especially when the weather is hot, by moving bare - bodied thereby exposing themselves more to malaria vector bites than the females.

Consistent with previous reports (Erhabor et al., 2007; Chikwem et al., 1997; Erhabor et al., 2012; Adewuyi, 2001), it was found that $P$. falciparum was the predominant species among plasmodium parasitized individuals. Plasmodium falciparum malaria may be associated with a potentially fatal outcome, particularly if there are delays in recognition and treatment (Abdullahi et al., 2009).

With regards to the economy, the age range between 21 and 45 years forms the bulk of the work force and therefore clinical disease in this group of individuals could lead to loss of manpower and thus have a negative economic impact on the country besides the adverse health effects.

\section{Conclusion}

The high prevalence of malaria parasitaemia among patients attending General Hospital, Minna, North Central Nigeria highlights the need for more efforts to be targeted at controlling malaria among individuals as this will ultimately lead to a reduction in the incidence of malaria in Minna and Nigeria at large.

\section{CONFLICT OF INTEREST}

The authors declare that they have no conflict of interest. 


\section{REFERENCES}

Abdullahi, A. U., Abubakar, T. \& Adamu, A. I. (2009). Malaria in Sokoto, North Western Nigeria. African Journal of Biotechnology, 8(24) 7,101-7105.

Adewuyi, J. O. (2001). The challenge of blood safety in Africa. Africa Sanguine, 4(2), 1-10.

Agboola, T. F., Ajayi, M. B., Adeleke, M. A., \& Gyang, P. V. (2010). Prevalence of malaria parasite among blood donors in Lagos University teaching hospital, Lagos Nigeria. Annals of Biological Research, 1, 72-75.

Badger-Emeka, L., Emeka, P., \& Egbu, V. (2013). Prevalence of Malaria Plasmodium Parasite among Blood Donors at Nsukka Area, Southeast Nigeria. BEST: International Journal of Humanities, Arts, Medicine and Sciences, 1, 45-50.

Cheesbrough, M. (2000). District Laboratory Practice Manual in Tropical Countries. Part 2. Cambridge University Press. Pp. 178-179.

Chikwem, J. O., Mohammed, I., Okara, G. C., Ukwandu, N. C., \& Ola. T.O. (1997). Prevalence of transmissible blood infections among blood donors at theUniversity of Maiduguri Teaching Hospital, Maiduguri, Nigeria. East African Medical Journal, 74, 213-216.

Ekwunife, C. A., Ozumba, N. A., Eneanya, C. I., \& Nwaorgu, O. C. (2011). Malaria infection among Blood Donors in Onitsha Urban, Southeast Nigeria. Sierra Leone Journal of Biomedical Research, 3(1), 21-26.

Epidi, T. T., Nwani, C. D., \&Ugorji, N. P. (2008). Prevalence of Malaria in Blood Donors in Abakaliki Metropolis, Nigeria. Scientific Research and Assay, 3, 162-164.

Erhabor, O., Azuonwu, O., \& Frank-Peterside, N. (2012). Malaria parasitaemia among long distance truck drivers in the Niger delta of Nigeria. African Health Science, 12, 98-103.

Erhabor, O., Ok, O., Awah, I., Uko, K. E. \& Charles, A. T. (2007). The prevalence of Plasmodia parasitaemia among donors in the Niger delta of Nigeria. Tropical Doctor, 37(1), 32-34.

Gillies, M. T., \& Coetzee, M. (1987). A supplement to the Anophelinae of Africa South of the Sahara (Afrotropical Region). Publications of the South African Institute for Medical Research, No. 55.

Goodman, C., Coleman, P. \& Mills, A. (2000). Economic analysis of malaria control in Sub-Saharan Africa. London School of Hygiene and Tropical Medicine, Mimeo.

Gray, J. C., Corran, P. H., Mangia, E., Gaunt, M. W., Li, Q., Tetteh, K. K., Polley, S. D., Conway, D. J., Holder, A. A., Bacarese- Hamilton, T., Riley, E. M., \& Crisanti, A. (2007). Profiling the antibody immune response against blood stage malaria vaccine candidates. Clinical Chemotherapy, 53, 124453.

Greenwood, B. M., \& Armstrong, J. R. (1991). Comparison of two simple methods for determining malaria parasite density. Transaction Royal Society of Tropical Medicine and Hygiene, 85, 186-188

Hay, S. I., Smith, D. L., \& Snow, R. W. (2008). Measuring malaria endemicity from intense to interrupted transmission. Lancet Infectious Disease, 8, 369-378.

Karunamoorthi, K., \& Sabesan, S. (2013). Insecticide Resistance in Insect Vectors of Disease with Special Reference to Mosquitoes: A Potential Threat to Global Public Health. Health Scope, 2, 4-18.
Mbanugo, J. I., \& Emenalo, S. (2004). Prevalence of Malaria Parasitaemia among Blood Donors in Owerri, Imo State, Nigeria. Nigerian Journal of Parasitology, 25, 75-80.

Muntaka, S., \& Opoku-Okrah, C. (2013). The Prevalence of Malaria Parasitaemia and Predisposition of ABO Blood Groups to Plasmodium falciparumMalaria among Blood Donors at a Ghanaian Hospital. AU Journal of Technology, 16, 255-260.

Sachs, J., \& Malaney, P. (2002). The economic and social burden of malaria. Nature, 415(6872), 680-685.

Sinka, M. E., Bangs, M. J., Manguin, S., Coetzee, M., Mbogo, C. M., Heminway, J., Patii, A. P., Temperley, W. H., Gething, P. W., Kabaria, C. W., Okara, R. M., Boeckel, T. V., Godfray, H. C. I., Harbach, R. E., \& Hay, S. I. (2010). The dominant Anopheles vectors of human malaria in Africa, Europe and the Middle East: occurrence data, distribution maps and bionomic précis. Parasites and Vectors, 3, 117.

Snow, R. W., \& Marsh, K. (2002). The consequences of reducing transmission of Plasmodium falciparum in Africa. Advance Parasitology, 52, 235-264.

Snow, R., Hay, S. I., Cox, J., Roggers, D. J., Randolph, S. E., Stern, D. I., Shanks, G. D., \& Myers, M. F (2005). The global distribution of clinical episodes of Plasmodium falciparum malaria. Nature, 434, 214-217.

Uneke, C. J., Ogbu, O., \&Nwojiji, V. (2006). Potential risk of induced malaria by blood transfusion in South-eastern Nigeria. McGill Journal of Medicine, 9(1), 8-13.

Wariso, K. T., \& Oboro, I. L. (2015). Prevalence of Plasmodiumparasitaemia among blood donors in port Harcourt, Nigeria, Advances in Microbiology, 5, 351-357.

White, M. T., Griffin, J. T., Churcher, T. S., Ferguson, N. M., Basanez, M. G., \& Gbani, A. C. (2011). Modelling the impact of vector control interventions on Anopheles gambiae population dynamics. Parasites and Vectors, 28, 4.

WHO (2000). Bench Aids for the Diagnosis of Malaria. World Health Organization, Geneva.

WHO (2011). World Malaria Report 2011. World Health Organization, Geneva. Available at http://www.who.int/malaria/publications/country/profile_gh_en .pdf.

WHO (2013). World Malaria Report 2013. World Health Organization, Geneva.

WHO (2014). World Malaria Report 2014. World Health Organization, Geneva. Available at http://www.who.int/entity/malaria/publications/world_malaria_r eport_2014/report/en/index.html.

Wongsrichanalai, C., Pickard, A., Wernsdorfer, W., \& Meshnick, S. (2002). Epidemiology of drug-resistant malaria. Lancet Infect Disease, 2, 209-18. 